Available online on 15.12.2019 at http://jddtonline.info
Open Access to Pharmaceutical and Medical Research
unrestricted non-commercial use, provided the original work is properly cited

Open $\overbrace{\text { Access }}$

Research Article

\title{
The effect of Bombyx mori larvae extract in reducing the toxicity of methotrexate in pregnant female albino rats
}

\author{
Nehad M. Ibrahim ${ }^{1}$, Samar Sayed ${ }^{1}$, Rania S. Ali ${ }^{1}$, Osama H. Ashour ${ }^{2}$, Ebtehal Mohammad Fikry ${ }^{3 *}$ \\ ${ }^{1}$ Comparative anatomy and embryology, Faculty of Science, Helwan University. Egypt \\ ${ }^{2}$ Biochemistry Department, National Organization for Drug Control and Research (NODCAR), Giza, Egypt \\ ${ }^{3}$ Pharmacology Department, National Organization for Drug Control and Research (NODCAR), Giza, Egypt
}

\begin{abstract}
Methotrexate (MTX) is an anticancer agent which is used alone or in combination with other anticancer agents in the treatment of breast cancer, epidermoid cancers of the head and neck, cutaneous $\mathrm{T}$ cell lymphoma, and lung cancer. Bombyx mori larvae have a huge value as health food especially for cardiac and diabetic patients, bronchial asthma, primary trigeminal neuralgia, vocal nodules and polyps and in the treatment of facial palsy and pain. In the present study meeting occurred under the normal conditions and about 40 pregnant female rats were used. Herein the pregnant females will be divided into five groups; group 1 will be used as negative control received distilled water, group 2 will used as positive control and recived buffer of Bombyx mori larvae extract, group 3 was treated with MTX at the $12^{\text {th }}$ day of gestation (at organogenesis phase), group 4 was treated with Bombyx mori larvae extract at $7^{\text {th }}, 9^{\text {th }}, 11^{\text {th }}, 13^{\text {th }}$ and $15^{\text {th }}$ days of gestation (during the oraganogensis period), \& group 5 was injected by MTX the $12^{\text {th }}$ day of gestation as well as bombyx mori larvae extract at $7^{\text {th }}, 9^{\text {th }}, 11^{\text {th }}, 13^{\text {th }}$ $\& 15^{\text {th }}$ days of gestation. Animals of all groups will be sacrificed at $20^{\text {th }}$ day, the end of gestation periods. Then livers of all pregnant rats were removed for examination. The obtained results showed decrease in maternal body weight gain plus increase in the abortion rate and uterine weight in the MTX-treated group. In addition, MTX induced an elevation in the examined liver oxidative stress biomarkers plus myeloperoxidase activity and decrease in reduced glutathione content and catalase activity; in mothers. Histopathological studies of liver tissues showed congestion of central vein of mothers in MTX group showing ballooning degeneration of hepatocytes, perivascular inflammatory cells infiltration and strong deposition of collagen fibers. Noteworthy, Bombyx mori larvae induced marked improvement in injuries associated with MTX administration.
\end{abstract}

Keywords: Methotrexate, Bombyx mori larvae, Pregnant female rats, Hepatotoxicity.

Article Info: Received 11 Oct 2019; Review Completed 21 Nov 2019; Accepted 29 Nov 2019; $\quad$ Available online 15 Dec 2019

Cite this article as:

Ibrahim MM, Sayed S, Ali RS, Ashour OH, Ebtehal MF, The effect of Bombyx mori larvae extract in reducing the toxicity of methotrexate in pregnant female albino rats, Journal of Drug Delivery and Therapeutics. 2019; 9(6-s):82-87 http://dx.doi.org/10.22270/jddt.v9i6-s.3772

ㅁ.

Ebtehal Mohammad Fikry, Department of Pharmacology, National Organization for Drug Control and Research, NODCAR, Giza, Egypt, P.O. BOX 29

\section{INTRODUCTION}

The hepatic injury mechanism has been studied broadly however it is still unclear. As exposed in preceding reports, the authors informed that mediation of lipid peroxidation might be the fundamental mechanism of serious hepatic reactions. Lipid peroxidation is one of the major ROS consequences which causing cell damage [1].

Noteworthy, Glutathione (GSH) as a major antioxidant and redox checker participate an important role in the defense against oxidants and electrophiles [2]. Accordingly, any mechanism which removes ROS or prevents hepatic GSH depletion or induce activation and production of GSH dependent enzymes may give protection for hepatotoxicity [3].
Chemotherapies are type of anti-cancer drug treatment. They work by killing cancer cells by moving throughout your body and are called a systemic treatment. It is also given to cancers with an elevated risk of micrometastatic disease [4].

Actually, Methotrexate (MTX), formerly known as amethopterin, is a chemotherapeutic agent and immune system suppressant. It is used to treat cancer and autoimmune diseases. It is used in some types of cancers for instance breast cancer, leukemia, lung cancer, lymphoma, and osteosarcoma. In addition, it's used to treat some types of autoimmune diseases such as psoriasis, rheumatoid arthritis, and Crohn's disease. MTX was made in 1947 and initially came into medical use to treat cancer, as it was less toxic than the then-current treatments [5].On the other hand, as with other chemotherapeutic agents, MTX exerts prominent oxidant effects in liver [6]. 
Of impact, natural products including crude extracts, bioactive components-enriched fractions, and pure compounds resultant from herbs plus herbal formulas have been proved to avoid and treat cancers [7].

Silkworms are larvae of the moth Bombyx mori, which is native to Asia, spins a cocoon of fiber that is the source of commercial silk. Bombyx morifeeds on the leaves of the mulberry tree and supplies as an excellent model structure because of its life cycle, it is cheap, and there are no ethical issues involved [8]. A thriving cocoon crop in sericulture depends mostly on healthy larval growth [9].

Larvae have been identified as a possible source of numerous chemical constituents, such as adipokinetic hormone, insulin-like growth factor-II, chymotrypsin inhibitors, b-N-acetyl glucosaminidase, DOPA, quinone amine conversion factor and sex pheromone bom-bykol have been accounted from the larvae $[10,11,12,13,14$, 15]. Interleukin-3 (IL-3) created from the silkworm is reported to be biologically identical to IL-3 produced from mammalian cells [16]. Processed larvae are used in particular diets for cardiac and diabetic patients because of their low cholesterol content [17]. The silkworm larvae can provide as a bioreactor for the production of low cost vaccines against different infectious diseases [16].

The propose of this assessment was to find out the effect of MTX exposure on pregnant albino rats and to assess whether these effects can be ameliorated by Bombyx mori larvae extract.

\section{MATERIAL AND METHODS:}

\section{I-Material:}

The white albino rat is one of the best species for experimental teratological studies. Its gestation period is short (about 21 day) and has large number of litters. Rats are characterized by their genetic stability and a very low rate of spontaneous malformation $[18,19,20,21,22]$. The time of the rat copulation is easily determined by vaginal smear examination method. Also, the numbers of corpora lutea of pregnancy can be counted easily and accurately [23] .The present experimental study complies with the guide for care and use of laboratory animals published by the US National Institutes of Health (NIH publication No. 85-23, revised 1996).

Females of 11-13 weeks old were selected for the present study and vaginal smears were prepared every morning and examined under the light microscope, according to the method of Snell [24], for 5 days to select the female with regular estrus. Two females with regular estrus cycle were selected in the pro-estrus stage and caged together with one male over night under controlled environmental conditions of temperature, humidity and light. The first day of gestation was determined by the presence of sperms in the vaginal smear [25].

\section{II-Methods}

\section{Experimental design}

Mating occurred under the normal conditions and about 40 pregnant female rats were divided into five groups $(n=6-$ 8, each) as follow:

-Group 1: Normal control group and was received distilled water.

-Group2: Buffer control group and was received buffer of Bombyx mori larvae extract.
-Group3: Methotrexate (14mg/Kg, once orally) was administered at the $12^{\text {th }}$ of gestation (at organogenesis phase) [26].

-Group4: Bombyx mori larvae extract $(45 \mathrm{mg} / \mathrm{kg}$, intramuscular) was given at 7 th $, 9^{\text {th }}, 11^{\text {th }}, 13^{\text {th }}$ and $15^{\text {th }}$ days of gestation (during the oraganogensis period) [27].

-Group5: Methotrexate + Bombyx mori larvae extract.

-Animals of all groups will be sacrificed at the end of gestation periods at $20^{\text {th }}$ day of gestation. Livers of all pregnant rats will be removed to be used in our study.

\section{Preparation of bombyx mori liposome}

Bombyx Mori adult larvae in the fifth age were collected, homogenized, filtered to obtain the homogenate. The homogenate then lyophilized to obtain crude dry extract of the larvae. From 2 grams of larvae we obtain $100 \mathrm{mg}$ powder.

Liposomal preparation was occurred, with slight modification, by mixing of $50 \mathrm{mg}$ of dry extract, $150 \mathrm{mg}$ cholesterol (Shaanxi Sangherb Bio-Tec, china), $500 \mathrm{mg}$ phosphatidyl choline (Shaanxi M.R Natural Product Co., Ltd., china) in $100 \mathrm{ml}$ chloroform until the mixture is completely dissolved. The mixture then was put in a rotary evaporator (lab first scientific, China) at temperature of $45 \mathrm{C}$ at a speed of $40 \mathrm{rpm}$. After complete evaporation the aqueous phosphate buffer pH 7 composed of $\mathrm{Na}_{2} \mathrm{HPO}_{4}, \mathrm{KH}_{2} \mathrm{PO}_{4}, \mathrm{NaCl}$, $\mathrm{KCl}$ was added to obtain total volume of 10 milliliter [28].

\section{1- Signs of toxicity:}

The following parameters were recorded and measured during gestation period or at scarification time:

a- The weight gain of pregnant rat: A daily record of the weight of the pregnant females was made throughout the whole gestation period.

b- The percentage of abortion: The percentages of abortion were calculated in each group; abortion was determined by the presence of blood drops and sudden drop in the weight of the pregnant females.

c- The uterine weights.

\section{2-Biochemical parameters:}

Determination of hepatic inflammatory marker; myeloperoxidase (MPO) activity:

Determination of MPO activity was done using a kinetic colorimetric method described by Bradley et al. [29].

Determination of hepatic oxidative stress biomarkers; contents of malondialdehyde (MDA) and reduced glutathione (GSH), and catalase (CAT) activity, as well as nitrite/nitrate index (NOx):

Liver contents of MDA and GSH, as well as catalase activity were performed according to manufacturer's prescripts using reagent kits (Biodiagnostic Company, Giza, Egypt). Additionally, vanadium trichloride was used to reduce nitrate to nitrite according to Miranda et al. [30] in nitric oxide assay. The method of nitrite estimation is based on Griess reaction that was performed using the kit provided by Biodiagnostic.

\section{3-Histological Examinations; stain with H\&E and Masson's trichrome:}

At $20^{\text {th }}$ day of gestation one lobe of half numbers of the liver of pregnant rats of all groups were fixed in $10 \%$ neutral formalin buffer for at least one week followed by washing 
with tap water. Then samples were processed using a graded ethanol series and embedded in paraffin. Paraffin sections were cut into $6 \mu \mathrm{m}$-thick slices and stained with hematoxylin and eosin for light microscopic examination. Additionally, other liver sections were stained with Masson's trichrome stain.

\section{4-Statistical analysis:}

Results were expressed as mean \pm SEM. Statistical analysis was carried out using one-way analysis of variance (ANOVA) followed by Tukey-Kramer Multiple Comparison Test. Probability values of less than 0.05 were considered statistically significant. Whereas the graphs were drawn using a prism computer program (GraphPad software Inc. V5, San Diego, CA) [31].

\section{RESULTS}

Effect of bombyx mori larvae extract, in pregnant rats exposed to MTX, on the body weight gain, the percentage of abortion, and the total uterine weight

Table 1 illustrates the effect of MTX as well as bombyx mori larvae on the body weight gain, the percentage of abortion, and the total uterine weight.
1- The effect on the body weight gain:

Here, we found that the weight gain of pregnant rats treated with MTX were decreased significantly by $53 \%$ and by $54 \%$ compared to normal control and buffer control groups; respectively. Conversely, bombyx mori larvae extract decreased MDA contents significantly by $138 \%$ and $104 \%$ as compared to MTX group in bombyx mori larvae group and bombyx mori larvae+MTX group; respectively.

\section{2- The effect on the percentage of abortion:}

In the current study, our results revealed that the percentage of abortion in control group was $17 \%$ while it was $25 \%$ in the MTX group and $17 \%$ in bombyx mori larvae+MTX group. The percentage of abortion was zero in buffer group and bombyx mori larvae+MTX group.

3- The effect on the total uterine weight:

The present study shows that the total uterine weight increased significantly by $22 \%$ and $18 \%$ compared to normal control and buffer control groups; respectively. While bombyx mori larvae decreased the total uterine weight significantly by $27 \%$ and $15 \%$ as compared to MTX group in bombyx mori larvae group and bombyx mori larvae+MTX group; respectively.

Table 1 represents the effects of treatment with bombyx mori larvae extract on the body weight gain, the percentage of abortion, and the total uterine weightof pregnant rats subjected to MTX.

\begin{tabular}{|c|c|c|c|}
\hline Groups & Weight gain (g) & Percentage of abortion & Total uterine weight (g) \\
\hline Control & $45.45 \pm 3.164$ & $17 \%$ & $35.72 \pm 0.470$ \\
\hline Buffer & $46.13 \pm 5.445$ & 0 & $36.93 \pm 1.656$ \\
\hline MTX & $21.25 \pm 2.270^{* !}$ & $25 \%$ & $43.63 \pm 1.910^{* !}$ \\
\hline Bom & $50.53 \pm 3.223^{\#}$ & 0 & $31.74 \pm 2.569^{\#}$ \\
\hline Bom + MTX & $43.43 \pm 3.472^{\#}$ & $17 \%$ & $36.92 \pm 1.657^{\#}$ \\
\hline
\end{tabular}

* vs Control group, ! vs Buffer group, \& \#vs MTX group (One-Way ANOVA followed by Tukey-Kramer multiple comparisons test; $\mathrm{p}<0.05)$. Where; Control: normal control, Buffer: buffer control, MTX: methotrexate, Bom: bombyx mori larvae.

\section{Effect of bombyx mori larvae extract on MPO activity and on oxidative stress biomarkers}

Figure 1 depicts the effect of bombyx mori larvae on MPO activity. Herein, administration of MTX increased MPO activity significantly by $203 \%$ and $280 \%$ as compared to normal control and buffer control groups; respectively. On the other hand, bombyx mori larvae extract decreased MPO activity significantly by $76 \%$ and $70 \%$ as compared to MTX group in bombyx mori larvae group and bombyx mori larvae+MTX group; respectively.

Figure 2 represents the effects of bombyx mori larvae on MDA content. Our results showed that MDA content was markedly elevated in MTX group by $37 \%$ and $34 \%$ compared to normal control and buffer control groups; respectively. Conversely, bombyx mori larvae extract decreased MDA contents significantly by $18 \%$ and $17 \%$ as compared to MTX group in bombyx mori larvae group and bombyx mori larvae+MTX group; respectively.

Figure 3 represents the effects of bombyx mori larvae on GSH content. The current study revealed that GSH content was obviously declined in MTX group by $29 \%$ and $36 \%$ compared to normal control and buffer control groups; respectively. In contrast, bombyx mori larvae extract augmented GSH contents significantly by $54 \%$ and $49 \%$ as compared to MTX group in bombyx mori larvae group and bombyx mori larvae+MTX group; respectively.

Figure 4 stands for the effects of bombyx mori larvae on catalase activity. The existing study disclosed that catalase activity was clearly decreased in MTX group by $56 \%$ and $45 \%$ compared to normal control and buffer control groups; respectively. On the contrary, bombyx mori larvae extract augmented catalase activities significantly by $80 \%$ and $103 \%$ as compared to MTX group in bombyx mori larvae group and bombyx mori larvae+MTX group; respectively. However, the activity of catalase was low to certain extent in buffer control and bombyx mori larvae groups as compared to normal control group by $20 \%$ and $21 \%$; respectively.

Figure 5 represents the effects of bombyx mori larvae on NOx content. The present study divulged that NOx content was apparently increased in MTX group by $26 \%$ and $19 \%$ compared to normal control and buffer control groups; respectively. In contrast, bombyx mori larvae extract augmented NOx contents significantly by $18 \%$ and $16 \%$ as compared to MTX group in bombyx mori larvae group and bombyx mori larvae+MTX group; respectively. 


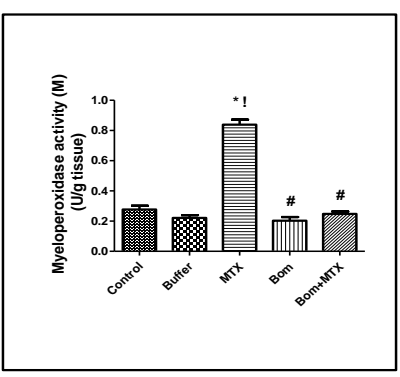

Figure 1

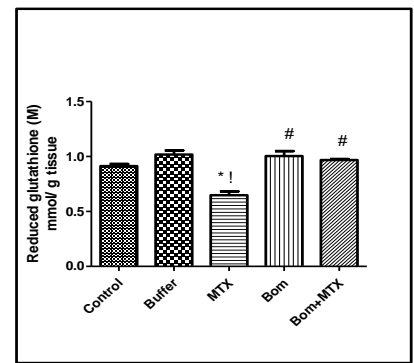

Figure 3

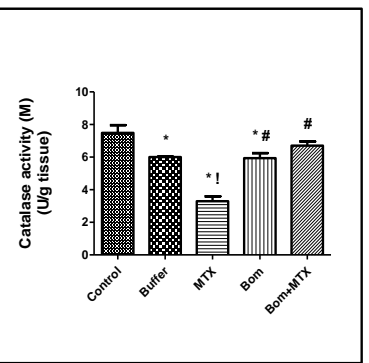

Figure 4

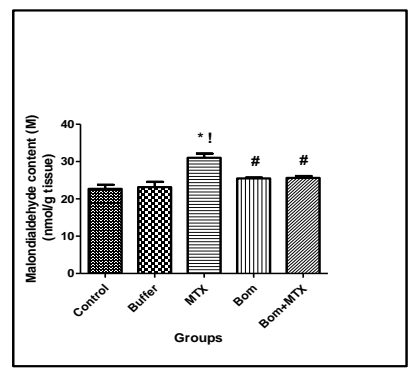

Figure 2

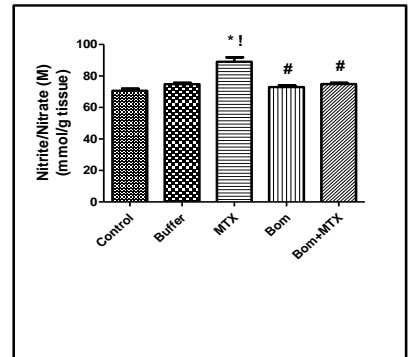

Figure 5

Figures 1,2,3,4, \&5 represents the effects of treatment with bombyx mori larvae extract on MPO, MDA, GSH, catalase \& NOx; respectively, in liver tissue of pregnant rats subjected to MTX.

Each bar with vertical line represents the mean \pm S.E.M of 6-8 rats per group. ${ }^{*} v s$ Control group, ! vs Buffer group, \& \#vs MTX group (One-Way ANOVA followed by Tukey-Kramer multiple comparisons test; p < 0.05). Where; Control: normal control, Buffer: buffer control, MTX: methotrexate, Bom: bombyx mori larvae.

\section{Effects of bombyx mori larvae extract on inflammation and fibrosis of the liver tissues}

1- As illustrated in Figures 6; normal histological structure of hepatic parenchyma in normal control group (A) and buffer control group (B). While MTX- treated group (C) demonstrated ballooning degeneration of hepatocytes (small arrow) and perivascular inflammatory cells infiltration (long arrow). Noteworthy, both group of bombyx mori larvae extract, either alone or co-administered with MTX, illustrated slight activation of Kupffer cells (arrow).
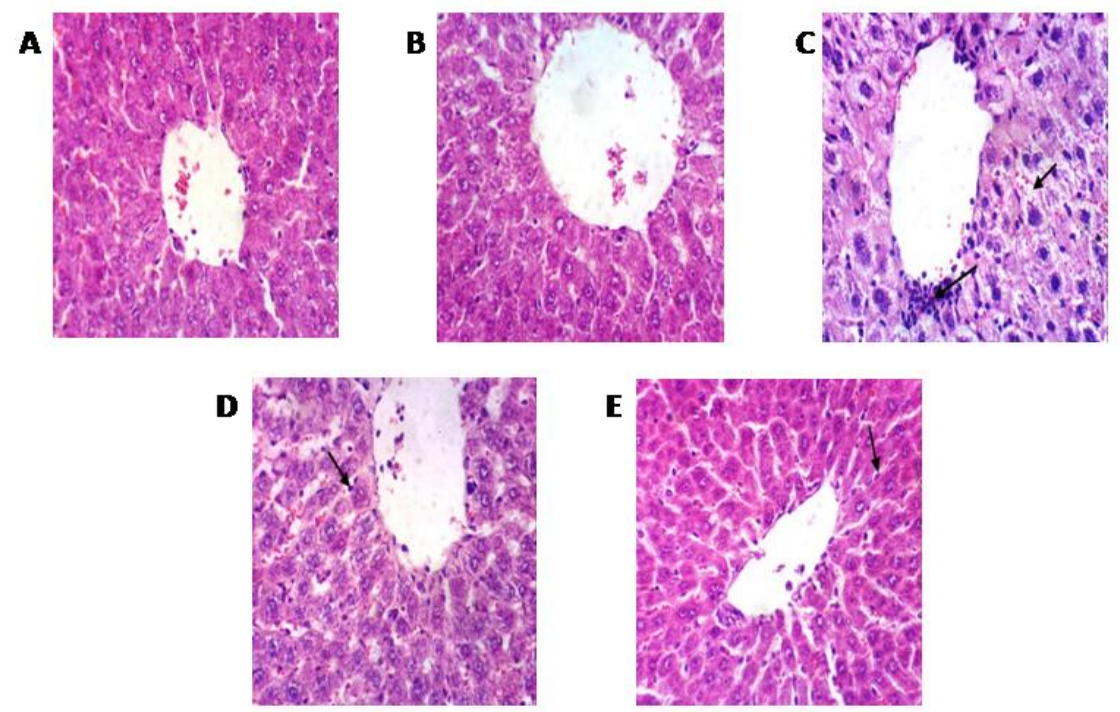

Figure 6 Effect of treatment with bombyx mori larvae extract on the histopathological examination in liver tissue subjected to MTX. Photomicrographs of mother liver sections stained with H\&E of normal control group (A) \& buffer group (B) showed normal histological structure of hepatic parenchyma. Whereas liver section of MTX group (C) showed ballooning degeneration of hepatocytes (small arrow) and perivascular inflammatory cells infiltration (long arrow), but both bombyx mori larvae extract treated groups (D) \& (E) showing slight activation of Kupffer cells (arrow); (H \& E X 400).

2- As demonstrated in Figures 7; there is normal weak histochemical reaction for collagen fibers in normal control group (A) and buffer control group (B). Whereas MTX-treated group (C) showed strong positive histochemical reaction for collagen fibers. On the other hand, both group of bombyx mori larvae extract, either alone or co-administered with MTX, illustrated weak histochemical reaction for collagen fibers. 

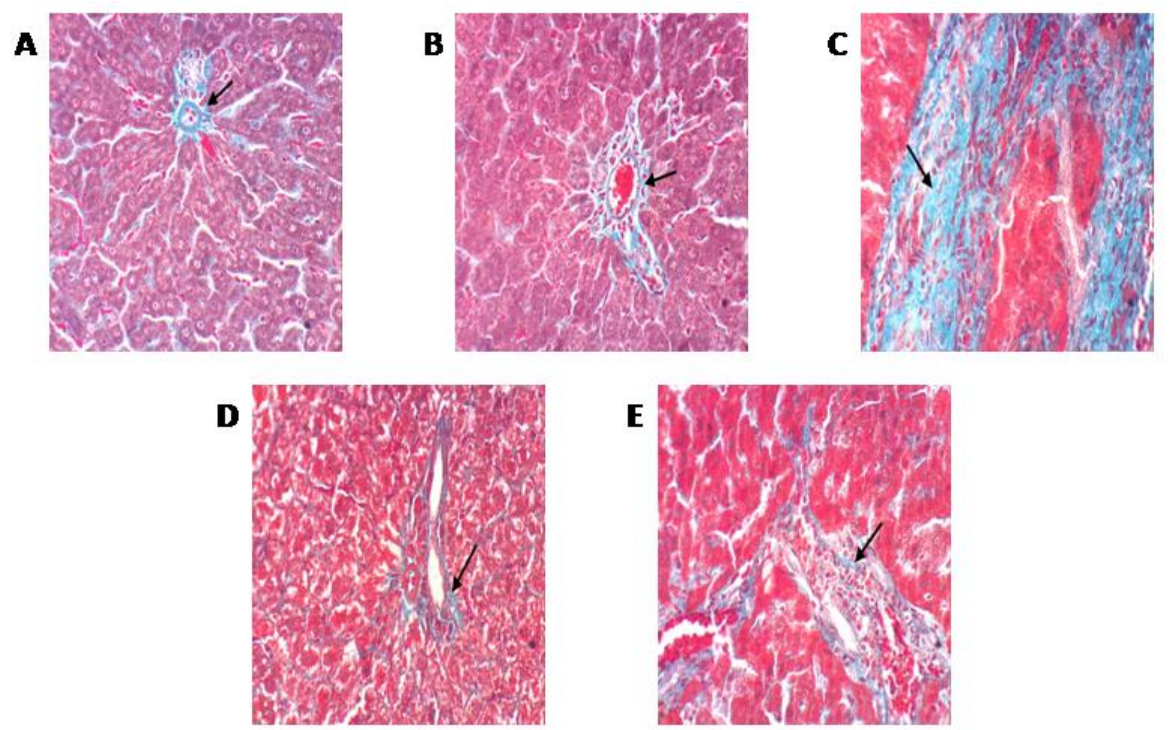

Figure 7 Effect of treatment with bombyx mori larvae extract on the fibrosis in liver tissue subjected to MTX. Photomicrographs of mother liver sections stained with Masson's Trichrome of normal control group (A) \& buffer group (B) showed normal weak histochemical reaction for collagen fibers. Whereas liver section of MTX group (C) showed strong positive histochemical reaction for collagen fibers, but both bombyx mori larvae extract treated groups (D) \& (E) showing weak histochemical reaction for collagen fibers; (Masson's Trichrome stain X 400).

\section{DISCUSSION}

This study investigated, for the first time, the advanced effect of Bombyx mori larvae extract in reducing the toxicity of MTX in pregnant female albino rats.

In the present study administration of MTX reduced the weight gain in pregnant rats exposed to MTX. Whereas weight gain is normal in Bombyx mori larvae+MTX group. MTX was shown to disturb food intake and induce intestinal hurt leading to deficient intestinal absorption and so body weight loss [32]. But Bombyx mori larvae keep the normal growth of the rats by improving the general condition by reducing cholesterol perhaps by increasing the anti oxidant status [33]. Really, larvae are used in special diets for cardiac and diabetic patients because of their low cholesterol content [34].

Regarding to the uterine weight in case of Bombyx mori larvae the results showed a significant reduction in the uterine weight which may be due to water extraction [35], while MTX group increased in the weight of uterus because MTX caused edema and this condition characterized by an overload of watery fluid collecting in the cavities or tissues of the body [36].

In fact, MTX in this work induced an oxidative stress revealed by significant reduction in liver GSH but it induced significant elevations in content of MDA and NOx, as well as MPO activity as compared to control animals. These achievements were in agreement with previous reports [37, 38,39 ] and these may be due to that MTX can inhibit some antioxidant enzymes which sequentially may cause lipid peroxidation to enlarge due to diminution in the activities of protective antioxidant enzymes such as catalase. Moreover, MTX inhibits cytosolic NADP-dependent dehydrogenase and NADP malic enzyme and leads to a decrease in intracellular NADPH levels. NADPH is crucial for glutathione reductase enzyme that maintains the levels of GSH, which is an important cytosolic antioxidant substance. Meanwhile, the reduction in GSH content may be due to the reduction of glutathione reductase activity so the reduction of oxidized glutathione to reduced glutathione is impaired [26].
Really, we found that Bombyx mori larvae administration caused elevation in GSH content and reduction in content of MDA and NOx, along with MPO activity. Moreover larvae improved the activity of protective antioxidant enzymes, catalase. According to $\mathbf{H u}$, et al. [40] the effects of larvae may be due to that larvae possesses potential radical scavenging properties. These are in agreement with the previous findings $[41,42]$.

In this study the livers of pregnant rat in MTX group showing ballooning degeneration of hepatocytes and perivascular inflammatory cells infiltration demonstrated using H\&E stain while, livers in Bombyx mori larvae+MTX group showing improvement in histopathological alterations. The nuclear changes may indicate decrease in cellular activity [43]. In addition, cellular infiltration was observed in this study which indicated inflammatory reaction.

Moreover, the current study revealed that strong deposition of collagen fibers in livers which were treated with MTX. According to Ohbayashi et al. [44] MTX induced proliferation of myofibroblasts, in an injured liver, progressed to deposition of collagen. On the other hand Bombyx mori larvae+MTX group showing weak reaction of collagen fibers. This result signifies the antifibrotic action of Bombyx mori larvae.

\section{ACKNOWLEDGMENT}

The authors are grateful to Dr. Kawkab Abdel-Aziz, Professor of Pathology, Faculty of Veterinary Medicine, Cairo University, for her helpful guide in histopathological examination.

\section{REFERENCES}

1- Lahneche A M, Boucheham R, Boubekri N, Bensaci S, Bicha S, Bentamenne A, \& Zama D. Sodium valproate-induced hepatic dysfunction in albino rats and protective role of n-Butanol extract of Centaurea sphaerocephala L. International Journal of Pharmacognosy and Phytochemical Res., 2017; 9(10):1335-1343.

2- Fernandez JC, Kaplowitz N.Hepatic mitochondrial glutathione transport and role in disease and toxicity. Toxicol. Appl. Pharmacol., 2005; 204: 263-273. 
3- Sabayan B, Foroughinia F,Chohedry A. A postulated role of garlic organosulfur compounds in prevention of valproic acid hepatotoxicity. Medical Hypotheses, 2007; 68: 512-514.

4- Perry, Michael J. The Chemotherapy source book. Philadelphia: Wolters Kluwer Health/Lippincott .Williams \& Wilkins, 2008; 978 0-7817-7328-7.

5- Sneader, Walter. Drug Discovery: A History. John Wiley \& Sons 2005; p. 251.

6- Vardi N, Parlakpinar H, Cetin A, Erdogan A, Cetin I . Protective effect of beta-carotene on methotrexate-induced oxidative liver damage. Toxicologic pathology, 2010; 38(4): 592-597.

7- Sanders K, Moran Z, Shi Z, Paul R, Greenlee H. Natural products for cancer prevention: Clinical Update 2016. Semin Oncol Nurs. 2016;32:215-240.

8- Hamamoto H, Tonoike A, Narushima K, Horie R, Sekimizu K. Silkworm as model organism to evaluate drug candidate toxicity and metabolism. Comparative Biochemistry and Physiology: Toxicology and Pharmacology, 2008; 149(3):334-339.

9- Khan MD, Saha BN. Growth and development of the mulberry silkworm, Bombyx mori L. on feed supplemented with alanine and glutamine. Sericolgia. 1995; 35:657-663.

10- Marumoto Y, Teruuchi T, Enjoh T, Numata F, Sakano K Purification and refolding of recombinant human IGF IIfrom silkworms infected with recombinant Bombyx morinuclear polyhedrosis virus. Biosci Biotech Biochem .,1992;56:13-16.

11- Ishibashi J, Kataoka H, Nagasawa H, Isogai A, Suzuki A.Isolation and identification of adipokinetic hormone of the silkworm Bombyx mori. Biosci Biotech Biochem., 1992; 56:66-70.

12- Jouni ZE, Wells MA. Purification and partial character-ization of a lutein-binding protein from the midgut of the silkworm, Bombyx mori. J Biol Chem., 1996; 271: 14722-14726.

13- Shinohara T, Aso Y, Shirai K, Fuji H, Funatsu G .Purifi-cation of Chymotrypsin inhibitors from larval hemolymphof the silkworm, Bombyx mori. Biosci Biotech Biochem., 1993; 57:1067-1071

14- Nagamatsu Y, Yanagisawa I, Kimoto M, Okamoto E, Koga I Purification of a chitooligosaccharidolytic b-Nacetylglucosaminidase from Bombyx morilarvae duringmetamorphosis and the nucleotide sequence of its cDNA Biosci Biotech Biochem., 1995; 59: 219-225

15- Aso Y, Yamamoto K, Yoshinaga T, Yamamoto H, Yamagami T. Partial purification and characterisation of DOPAQuinone Imine conversion factor from larval hemolymphof the silkworm, Bombyx mori. Biosci Biotech Biochem ,1995;59:277-281.

16- Datta RK . Silkworms to produce human vaccine. Indian Silk, 1994:33: 33-35.

17- De Z. Treatment of bronchial asthma with Ningxiao Drink in 85 patients. Shandong J Trad Chin Med., 1991;10:23.

18- Hai H .Treatment of 376 cases of male infertility with Zengjing pill Jiangxi J Trad Chin Med., 1989; 20: 10-11.

19- TuchmannH. Methods for evaluating teratognic properties of new drugs. In:"Methods in Drug Evaluation." North-Holand publishing company, 1966

20- Banerjee BN, Durlopo RS.Incidence of teratological anomalies controls charles River C-D strain rats. Toxico., 1973; 1: 151-154.

21- Wilson JG. Mechanisms of teratogenisis. Am. J. Anat., 1973;136(2): 129-131.

22- Tuchmann H. Drug effect on the fetus: General principles of drug induced congenital abnormalities. ADIS Press, 1977; 39.

23- Edwards JA. The external development of the rabbit and rat embryo In "Advances in Teratology", 3, Woollham, D.H.M.; Ed., Academic Press, 1968; 239.

24- Snell GD.Biology of the laboratory Mouse, 5th ed .The Blakiston company, philadeliphia., 1956.
25- McClain RM, BeckerB A. Teratogenicity, foetal toxicity and placental transfer of lead nitrate in rats. Toxicol. App.l Pharmacol., 1975;(1): 72-82.

26- Coleshowers CL, Oguntibeju 00, Ukpong M, Truter EJ .Effects Of Methotrexate On Antioxidant Enzyme Status In A Rodent Model. Medical Technology SA., 2010; 24 (1): 5-9.

27- Cho MR, Choue R, Chung SH, Ryu JW. Effect of silkworm powders on blood glucose and blood lipid concentrations of non-insulin dependent (Type 2) diabetes patients. Korean J. Nutrition, 1998; 31: 1139-1150.

28- Nounou MM, El-Khordagui LK, Khalafallah N. Effect of various formulation variables on the encapsulation and stability of dibucaine base in multilamellar vesicles. Acta Pol. Pharm, $2005 ; 62,369-379$.

29- Bradley PP, Priebat DA, Christensen RD, Rothstein G. Measurement of cutaneous inflammation: estimation of neutrophil content with an enzyme marker.Journal of Investigative Dermatology, 1982; 78(3):206-209.

30- Miranda KM, Espey MG, Wink DA.Arapid simple spectrophotometric method for simultaneous detection of nitrate and nitrite. Nitric Oxide, 2001; 5 (1): 62-71.

31- Armitage P, Berry G.Comparison of several groups. In: Blackwell Scientific Publications, 1987;186-213.

32- de Koning BA, van Dieren JM, Lindenbergh DJ, van der Sluis M Matsumoto $\mathrm{T}$, Yamaguchi $\mathrm{K}$, et al.contributions of mucosa immune cells to methotrexate-induced mucositis. International immunology, 2006; 18(6):941-949.

33- Tajuddin M, Ahmad NN. Effect of Unani formulation on lipid profile in rat. Indian J Pharmacol., 2006;38:56-7.

34- Ramakanth, Raman KVA .Cocoon Pelade for betterhealth. Indian Silk., 1997; 35: 35.

35- Mao XJ, Mao XP, Xiao QC, Jiang Q, Yang XY. Pharmacological study of antifertility on Bombyx batryticatus. J. Yunnan Coll. Tradit. Chin. Med., 2002;25: 26-28.

36- Iikuni N, Iwami S, Kasai S, Tokuda H. Intern Med., 2004 ;43(9):846 51

37- Cetin A, Kaynar L, Kocyigit I, et al. Role of grape seed extract on methotrexate induced oxidative stress in rat liver. The American Journal of Chinese Medicine., 2008;36(5):861-872.

38- KimHJ, LeeWH,YoonCH, Jeong JC, Nam KS, Kim HM,ChooYK, Lee $\mathrm{MC}, \mathrm{KimCH}$. Bombycis corpus extract prevents amyloid-betainduced cytotoxicity and protects superoxide dismutase activity in cultured rat astrocytes. Pharmacol. Res., 2001; 43:11-16.

39- Vardi N, Parlakpinar H, Cetin A, Erdogan A, Ozturk IC. Protective effect of $\beta$-carotene on methotrexate-induced oxidative liver damage. Toxicologic Pathology., 2010; 38(4):592-597.

40- Hu MB, Wang J L, Liu Y J, Yuan X, Li J H, Wu CJ, Li L. Structure characterization and antioxidant properties of proteins extracted from the larva of Bombyx mori L. Tropical Journal of Pharmaceutical Res., 2018; 17(11).

41- Zhao Q, Huo LQ, Jia TZ. Effects of Different Processing Methods on in vitro Antioxidant Activity and Inhibiting Capacity for Tyrosinase of Bombyx batryticatus. Chin. J. Exp. Tradit. Med. Form, 2014; 20: 17-23.

42- Xing DX, Liao ST, Luo GQ, Li L, Xiao Y, Li QR, Ye MQ, Yang Q.Extraction process optimization and antioxidant activity determination of polysaccharide from Bombyx batryticatus. Sci. Seri., 2015; 41: 1088- 1093.

43- Cetinkaya A, Bulbuloglu E ,Kurutas EB, Kantarceken B. Nacetylcysteine ameliorates methotrexate-induced oxidative liver damage in rats. Medical science monitor., 2006; 12(8): 274-278.

44- Ohbayashi M, Suzuki M, Yashiro Y, Fukuwaka S, Yasuda M, Kohyama N, Kobayashi Y, Yamamoto T. Induction of pulmonary fibrosis by methotrexate treatment in mice lung in vivo and in vitro. The Journal of toxicological sciences, 2010;35(5): 653-661. 